\title{
Ligaments of the Lisfranc joint in MRI: 3D-SPACE (sampling perfection with application optimized contrasts using different flip-angle evolution) sequence compared to three orthogonal proton-density fat-saturated (PD fs) sequences
}

\author{
Ulbrich, Erika J ; Zubler, Veronika ; Sutter, Reto ; Espinosa, Norman ; Pfirrmann, Christian W ;
} Zanetti, Marco

\begin{abstract}
PURPOSE: To compare the detection rate and visibility of the ligaments in the Lisfranc joint with a single 3D (-SPACE) MR sequence and three orthogonal PD fat-saturated sequences. MATERIALS AND METHODS: Thirty-one asymptomatic feet and 15 patients with posttraumatic pain in the Lisfranc joint were evaluated with a 3D-SPACE-sequence $(0.5 \mathrm{~mm}$ section thickness, acquisition time 10:22 min, secondary reformations) and three orthogonal PD fs sequences (2 mm section thickness, 9:20 min). The Lisfranc-ligament, the dorsal and plantar tarsometatarsal ligaments (TMT), the dorsal, interosseous, and plantar intermetatarsal ligaments (IMT) (24 ligaments for each foot) were assessed. RESULTS: In asymptomatic feet, 692 ligaments were detected with the SPACE sequence, thereof $90.6 \%$ exhibited normal signal, and most $(96.9 \%)$ were completely visible on one single image. A total of 659 ligaments were detected with the PD fs sequence, thereof $86.6 \%$ yielded normal signal, and $28.5 \%$ were completely visible on one single image. In patients, 327 ligaments were detected with SPACE, thereof $50.6 \%$ appeared completely visible with high signal. On PD fs, 308 ligaments were detected, $42.2 \%$ of the ligaments had high signals. CONCLUSIONS: The ligaments of the Lisfranc joint are better detected with a single 3D-SPACE sequence and secondary reformations than with three orthogonal PD fs sequences.
\end{abstract}

DOI: https://doi.org/10.1007/s00256-012-1491-5

Posted at the Zurich Open Repository and Archive, University of Zurich

ZORA URL: https://doi.org/10.5167/uzh-64832

Journal Article

Published Version

Originally published at:

Ulbrich, Erika J; Zubler, Veronika; Sutter, Reto; Espinosa, Norman; Pfirrmann, Christian W; Zanetti, Marco (2013). Ligaments of the Lisfranc joint in MRI: 3D-SPACE (sampling perfection with application optimized contrasts using different flip-angle evolution) sequence compared to three orthogonal protondensity fat-saturated (PD fs) sequences. Skeletal Radiology, 42(3):399-409.

DOI: https://doi.org/10.1007/s00256-012-1491-5 


\title{
Ligaments of the Lisfranc joint in MRI: 3D-SPACE (sampling perfection with application optimized contrasts using different flip-angle evolution) sequence compared to three orthogonal proton-density fat-saturated (PD fs) sequences
}

\author{
Erika J. Ulbrich • Veronika Zubler • Reto Sutter • \\ Norman Espinosa • Christian W. Pfirrmann • \\ Marco Zanetti
}

Received: 18 June 2012 / Revised: 11 July 2012 / Accepted: 12 July 2012 /Published online: 23 August 2012

(C) ISS 2012

\begin{abstract}
Purpose To compare the detection rate and visibility of the ligaments in the Lisfranc joint with a single 3D (-SPACE) MR sequence and three orthogonal PD fat-saturated sequences.

Materials and methods Thirty-one asymptomatic feet and 15 patients with posttraumatic pain in the Lisfranc joint were evaluated with a 3D-SPACE-sequence $(0.5 \mathrm{~mm}$ section thickness, acquisition time 10:22 min, secondary reformations) and three orthogonal PD fs sequences ( $2 \mathrm{~mm}$ section thickness, 9:20 min). The Lisfranc-ligament, the dorsal and plantar tarsometatarsal ligaments (TMT), the dorsal, interosseous, and plantar intermetatarsal ligaments (IMT) (24 ligaments for each foot) were assessed.

Results In asymptomatic feet, 692 ligaments were detected with the SPACE sequence, thereof $90.6 \%$ exhibited normal signal, and most (96.9\%) were completely visible on one single image. A total of 659 ligaments were detected with
\end{abstract}

\section{E. J. Ulbrich $(\bowtie)$}

Department of Diagnostic and Interventional Radiology,

University Hospital Zürich,

Rämistr. 100, 8091 Zürich, Switzerland

e-mail: erikajulbrich@googlemail.com

V. Zubler $\cdot$ R. Sutter $\cdot$ C. W. Pfirrmann $\cdot$ M. Zanetti

Department of Radiology,

Orthopedic University Hospital Balgrist,

Forchstr. 340, 8008 Zürich, Switzerland

N. Espinosa

Department of Orthopedic Surgery,

Orthopedic University Hospital Balgrist,

Forchstr. 340, 8008 Zürich, Switzerland the PD fs sequence, thereof $86.6 \%$ yielded normal signal, and $28.5 \%$ were completely visible on one single image.

In patients, 327 ligaments were detected with SPACE, thereof $50.6 \%$ appeared completely visible with high signal. On PD fs, 308 ligaments were detected, $42.2 \%$ of the ligaments had high signals.

Conclusions The ligaments of the Lisfranc joint are better detected with a single 3D-SPACE sequence and secondary reformations than with three orthogonal PD fs sequences.

Keywords Lisfranc joint - Ligaments · MRI · SPACE · PD fs sequence · Pain in the Lisfranc joint

\section{Introduction}

Midfoot sprains with concomitant injury of the tarsometatarsal (TMT) joints are typical sequela after sports injuries. There is a wide spectrum of injuries ranging from partial sprains with no displacement to complete tears with frank diastasis as well as complex fracture-dislocations [1-5]. The Lisfranc joint (also the tarsometatarsal joint) is a multifaceted polyarticular system between the distal row of the tarsal bones and the five metatarsal bones [6-9]. The joint complex is stabilized by the articular capsules, ligaments, and tendons [6-8]. Due to the complex anatomy, the identification of specific structures becomes difficult. However, proper identification of the extent of ligament and osseous injuries by means of imaging procedures is of importance to get the correct diagnosis and the appropriate treatment plan [10-14].

The Lisfranc joint ligament system consists of the cuneometatarsal ligaments (these are usually called "Lisfranc 
ligaments"), the tarsometatarsal (TMT) ligaments (dorsal and plantar), and the intermetatarsal (IMT) ligaments (dorsal, interosseous, and plantar) [8].

Several studies have focused on morphology of some of the ligaments pertaining to the Lisfranc joint [7, 15-21]. To our knowledge, only one cadaver study addressed in detail the complete intricate Lisfranc ligament anatomy [15]. New 3D sequences with secondary reformations have been successfully applied in different anatomical regions [22-25], and the MR acquisition times are feasible for clinical application. Secondary reformations have the potential for better visibility of oblique-running structures like the ligaments about the Lisfranc joint. While earlier studies using 3Dgradient echo sequences had the disadvantage of a more hyperintense signal in normal ligaments compared to spinecho sequences, recently $3 \mathrm{D}$ releases of turbo-spin echo sequences became available, called SPACE (Siemens Healthcare, Forchheim, Germany) or CUBE (GE Medical Systems, Solingen, Germany). These sequences employ thin-sections for secondary reformations with similar signal characteristics as conventional turbo-spin echo sequences, which may be useful for the evaluation of obliquely oriented ligaments. To our knowledge, the 3D-SPACE or CUBE sequence has been evaluated only for the ankle [26] but not for other areas in the foot.

The aim of this study was to compare the detection and visibility of the ligaments in the Lisfranc joint with a 3DSPACE MR sequence and three orthogonal PD fs sequences in patients and asymptomatic feet.

\section{Materials and methods}

This prospective study was approved by the ethics committee and all patients and healthy volunteers provided written informed consent.

\section{Subjects}

Thirty-one volunteers (mean age 31.4 years, range $22-$ 64 years) with asymptomatic feet were enrolled for the study. Subjects were excluded if any of the following aspects was present: history of foot trauma (e.g., fracture or ligament rupture at the midfoot), previous foot surgery, systemic disease (rheumatic disorder, diabetes, neoplasia), or contraindications to perform MRI (e.g., pacemaker, pregnancy, claustrophobia).

Fifteen consecutive patients (mean age 51.2 years, range 23-88 years) suffering from midfoot pain suspicious for fracture or ligament rupture within the Lisfranc joint were included into the study. A fellowship-trained orthopedic foot and ankle surgeon with at least 5 years of experience in his field examined all patients clinically. Twelve patients had an acute trauma and midfoot pain, four of them had a fracture of the Lisfranc joint (confirmed by computed tomography). Two of the patients with fracture were operated on. We included three patients with chronic midfoot pain.

\section{MR acquisition}

MR images of the foot were obtained using a 3-T MR scanner (MAGNETOM Verio, Siemens Medical Solution, Erlangen, Germany). Parameters for the transversely acquired 3D-SPACE (Sampling Perfection with Application optimized Contrasts using different flip-angle Evolution) T2-weighted sequence and for the three orthogonal standard PD (proton-density) fat-saturated sequences are illustrated in Table 1. All images were acquired with a dedicated 15channel knee coil with the patient in prone position. The foot was padded out additionally with pillows to reduce motion artefacts in the coil.

Image analysis

All MR images were evaluated independently by two radiologists with 5 years of experience in musculoskeletal imaging (EJU, VZ) who were blinded to the clinical data. Before the readout started, the first reader evaluated five random asymptomatic feet (two males, three females, mean age 36.2 years, range 27 to 50 years) to define the characteristic normal signal of all the ligaments (Table 2). Three weeks later, the first reader analyzed all subjects. The second reader analyzed one-third of all subjects. The data of the second reader were used for interobserver data. The images were analyzed on the Philips Extended Brilliance 190P workstation, using 3D-reformats for the 3D-SPACE sequence. In accordance with the standard foot anatomy text book by Sarrafian et al. [8], 24 ligaments in the Lisfranc joint were analyzed $(\mathrm{C}=$ Cuneiform, $\mathrm{Cub}=$ Cuboid, $\mathrm{M}=$ Metatarsal, $\mathrm{p}=$ plantar, $\mathrm{d}=$ dorsal):

The Lisfranc ligament (pC1-M2), seven dorsal tarsometatarsal (TMT) ligaments (dC1-M1, dC1-M2 (= dorsal Lisfranc ligament), dC2-M2, dC3-M2, dC3-M3, dCub-M4, dCub-M5), six plantar tarsometatarsal ligaments (pC1-M1, $\mathrm{pC} 1-\mathrm{M} 2,3$ (= plantar Lisfranc ligament), pC2-M2, pC3M3,4, pCub-M4, pCub-M5), and ten intermetatarsal (IMT) ligaments (containing of three dorsal intermetatarsal ligaments, four interosseous intermetatarsal ligaments and three plantar intermetatarsal ligaments) (Figs. 1, 2, 3, and 4). All ligaments were assessed concerning the overall detection and the visibility of the entire course of the ligament on one single image. If the ligament was detectable, the appearance of the ligament was assessed using a five-point score: $1=$ completely visible indicating that the entire course of the ligament is visible on one single image with normal signal, 
Table 1 Parameters of the MR protocol

\begin{tabular}{lllll}
\hline Acquisition direction & $\begin{array}{l}\text { PD fs } \\
\text { Trans }\end{array}$ & $\begin{array}{l}\text { PD fs } \\
\text { Sag }\end{array}$ & $\begin{array}{l}\text { PD fs } \\
\text { Cor }\end{array}$ & $\begin{array}{l}\text { SPACE } \\
\text { Trans }\end{array}$ \\
\hline TR (ms) & 2,070 & 2,070 & 2,250 & 1,300 \\
TE (ms) & 38 & 38 & 40 & 36 \\
FOV (mm) & $180 \times 180$ & $180 \times 180$ & $120 \times 120$ & $180 \times 180$ \\
Matrix size & $448 \times 336$ & $448 \times 336$ & $384 \times 288$ & $320 \times 288$ \\
Slice thickness $(\mathrm{mm})$ & 2 & 2 & 2 & 0,5 \\
Voxel size $\left(\mathrm{mm}^{3}\right)$ & $0.5 \times 0.4 \times 2$ & $0.5 \times 0.4 \times 2$ & $0.4 \times 0.3 \times 2$ & $0.6 \times 0.6 \times 0.5$ \\
Flip angle $\left({ }^{\circ}\right)$ & 180 & 180 & 180 & PDvar \\
Averages & 1 & 1 & 2 & 1 \\
Bandwidth $(\mathrm{Hz} /$ pixel) & 151 & 151 & 151 & 434 \\
Echo train time (ms) & 41 & 41 & 21 & 229 \\
Echo spacing (ms) & 12.7 & 12.7 & 13.5 & 5.2 \\
Echo train length & 7 & 7 & 7 & 70 \\
PAT mode & 2 & 2 & 2 & PAT2 \\
Slices & 29 & 29 & 29 & 176 \\
Time (min:s) & 3 & 3 & $3: 20$ & $10: 22$ \\
\hline
\end{tabular}

Table 2 Summary of signal characteristics of Lisfranc ligaments

\begin{tabular}{|c|c|c|c|}
\hline Ligament groups & Ligaments & Ligaments abbreviation & Signal characteristic* \\
\hline Interosseous TMT & Interosseous Lisfranc Lig & iTMT pC1-M2 & Low \\
\hline \multirow[t]{7}{*}{ Dorsal TMT } & Dorsal tarsometatarsal Lig & dTMT dC1-M1 & Low \\
\hline & Dorsal Lisfranc Lig & dTMT dC1-M2 & Muscle-isointense \\
\hline & Dorsal tarsometatarsal Lig & dTMT dC2-M2 & Low \\
\hline & Dorsal tarsometatarsal Lig & dTMT dC3-M2 & Muscle-isointense \\
\hline & Dorsal tarsometatarsal Lig & dTMT dC3-M3 & Low \\
\hline & Dorsal tarsometatarsal Lig & dTMT dCub-M4 & Low \\
\hline & Dorsal tarsometatarsal Lig & dTMT dCub-M5 & Low \\
\hline \multirow[t]{6}{*}{ Plantar TMT } & Plantar tarsometatarsal Lig & pTMT pC1-M1 & Low \\
\hline & Plantar Lisfranc Lig & pTMT pC1-M2,3 & Muscle-isointense, striated \\
\hline & Plantar tarsometatarsal Lig & pTMT pC2-M2 & Low \\
\hline & Plantar tarsometatarsal Lig & pTMT pC3-M3,4 & Low \\
\hline & Plantar tarsometatarsal Lig & pTMT pCub-M4 & Low \\
\hline & Plantar tarsometatarsal Lig & pTMT pCub-M5 & Low \\
\hline \multirow[t]{3}{*}{ Dorsal IMT } & Dorsal intermetatarsal Lig & dIMT2-3 & Low \\
\hline & Dorsal intermetatarsal Lig & dIMT3-4 & Low \\
\hline & Dorsal intermetatarsal Lig & dIMT4-5 & Low \\
\hline \multirow[t]{4}{*}{ Interosseous IMT } & Interosseous intermetatarsal Lig & iIMT1-2 & Muscle-isointense \\
\hline & Interosseous intermetatarsal Lig & iIMT2-3 & Muscle-isointense \\
\hline & Interosseous intermetatarsal Lig & iIMT3-4 & Muscle-isointense \\
\hline & Interosseous intermetatarsal Lig & iIMT4-5 & Muscle-isointense \\
\hline \multirow[t]{3}{*}{ Plantar IMT } & Plantar intermetatarsal Lig & pIMT2-3 & Low to Muscle-isointense \\
\hline & Plantar intermetatarsal Lig & pIMT3-4 & Low to Muscle-isointense \\
\hline & Plantar intermetatarsal Lig & pIMT4-5 & Low to Muscle-isointense \\
\hline
\end{tabular}

* On PD fs sequence and SPACE sequence $d$ dorsal, $i$ interosseous, $p$ plantar, TMT tarsometatarsal, IMT intermetatarsal, $C 1$ first cuneiform, $C 2$ second cuneiform, $C 3$ third cuneiform, $C u b$ cuboid, $M 1$ first metatarsal, M2 second metatarsal, M3 third metatarsal, M4 fourth metatarsal, M5 fifth metatarsal 
Fig. 1 Lisfranc ligaments. a Diagram of the dorsal (white arrowhead), interosseous (white arrow), and plantar (black arrowhead) Lisfranc ligament connecting the first cuneiform with the basis of the second metatarsal, illustrated on a three-dimensional view (left), on a transverse view (right top), and on a coronal view (right bottom). b Dorsal Lisfranc ligament (dTMT dC1-M2), transverse view, with muscleisointense appearance. c Dorsal Lisfranc ligament (dTMT dC1M2), coronal view, with muscle-isointense appearance. d Interosseous Lisfranc ligament (iTMT pC1-M2), transverse view, with low signal. e Interosseous Lisfranc ligament (iTMT pC1-M2), coronal view, with low signal. f Plantar Lisfranc ligament (pTMT dC1M2,3), transverse view, with striated, muscle-isointense appearance. The ligament connects the plantar side of the first cuneiform with the bases of the second and third metatarsals. $\mathbf{g}$ Plantar Lisfranc ligament (pTMT dC1-M2,3), coronal view with striated, muscleisointense appearance. The ligament connects the plantar side of the first cuneiform with the bases of the second and third metatarsals. Note: SPACE sequence is left and PD fs sequence is right in Fig. 1b-g
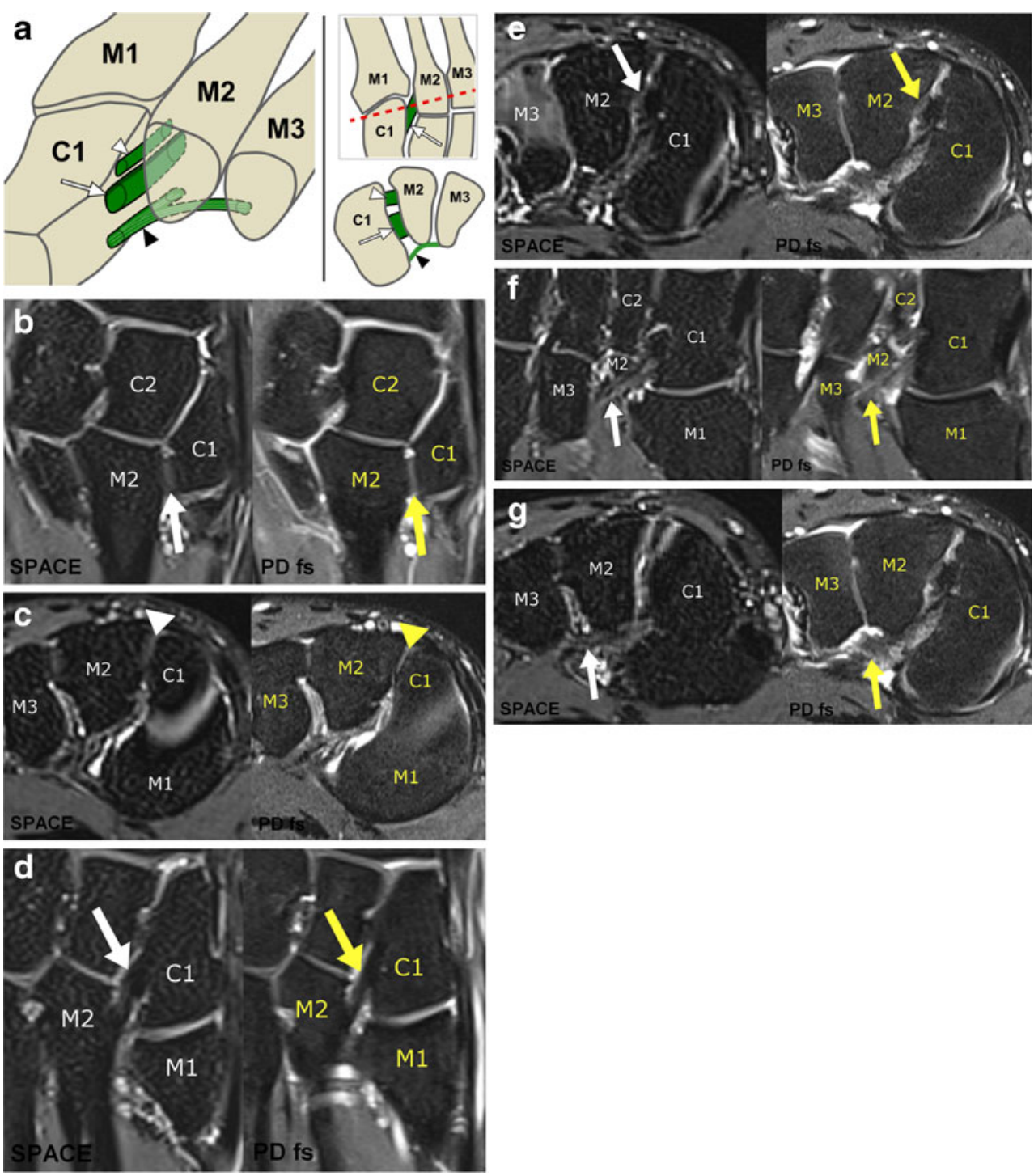

$2=$ partially visible with normal signal, $3=$ completely visible with high signal, $4=$ partially visible with high signal, $5=$ absent.

\section{Lisfranc joint ligament assessment}

The following ligaments were assessed:

1. Lisfranc ligaments: The Lisfranc ligament (pC1-M2) is the first and thickest interosseous cuneometatarsal ligament (medial) of three interosseous ligaments corresponding to the first, second, and third cuneometatarsal spaces. The Lisfranc ligament runs obliquely outward and slightly downward from the lateral surface of the first cuneiform $(\mathrm{pC} 1)$ to the lower half of the medial surface of the second metatarsal base (M2) (Fig. 1). The oblique-running dorsal Lisfranc ligament (dC1-M2) is the thinnest Lisfranc ligament and is part of the dorsal TMT ligaments. The most plantar Lisfranc ligament ( $\mathrm{pC} 1-\mathrm{M} 2,3)$ has the worst visibility of all cuneometatarsal ligaments, features a striated appearance, and is part of the plantar TMT ligaments (Fig. 1).

2. Dorsal tarsometatarsal (TMT) ligaments: There are seven dorsal TMT ligaments (dC1-M1, dC1-M2 (= dorsal Lisfranc ligament), dC2-M2, dC3-M2, dC3-M3, dCubM4, dCub-M5) connecting the cuneiforms and the cuboid with the metatarsal bases (Fig. 2). Five of them (dC1-M1, dC2-M2, dC3-M3, dCub-M4, dCub-M5) have a straight course. Two dorsal TMT ligaments (dC1-M2, dC3-M2) run obliquely from the base of the second metatarsal to the dorsal side of the first $(\mathrm{dC} 1$ M2 = dorsal Lisfranc ligament) and third cuneiforms (dC3-M2). The ligaments that connect the bases of the fourth and fifth metatarsals with the cuboid are often missing (dCub-M4, dCub-M5).

3. Plantar TMT ligaments: The plantar ligaments are larger than the dorsal ones and four of them ( $\mathrm{pC} 1-\mathrm{M} 1, \mathrm{pC} 2-\mathrm{M} 2$, pCub-M4, pCub-M5) connect the cuneiforms and the 
Fig. 2 Tarsometatarsal

ligaments. a Diagram. b

Example of a straight-running dorsal (closed arrowhead) and plantar (open arrowhead) TMT ligament of the first TMT with low signal appearance (dTMT dC1-M1; pTMT pC1-M1), sagittal view, between the first cuneiform and the basis of the first metatarsal. c dTMT dC3-M2, transverse view, obliquerunning ligament between the dorsal side of the third cuneiform and the basis of the second metatarsal with muscle-

isointense appearance. $\mathbf{d}$ dTMT dC3-M2, sagittal view, obliquerunning ligament between the dorsal side of the third cuneiform and the basis of the second metatarsal with muscleisointense appearance. In the $\mathrm{PD}$ fs sequence, this ligament is not visible because no 3D reconstruction can be performed. e pTMT $\mathrm{pC} 3-\mathrm{M} 3,4$, transverse view, oblique-running ligament between the plantar side of the third cuneiform and the bases of the third and fourth metatarsal with low signal appearance. $\mathbf{f}$ pTMT pC3-M3,4, sagittal view, oblique-running ligament between the plantar side of the third cuneiform and the bases of the third and fourth metatarsal with low signal appearance. Note: SPACE sequence is left and $\mathrm{PD}$ fs sequence is right in Fig. 2b-f
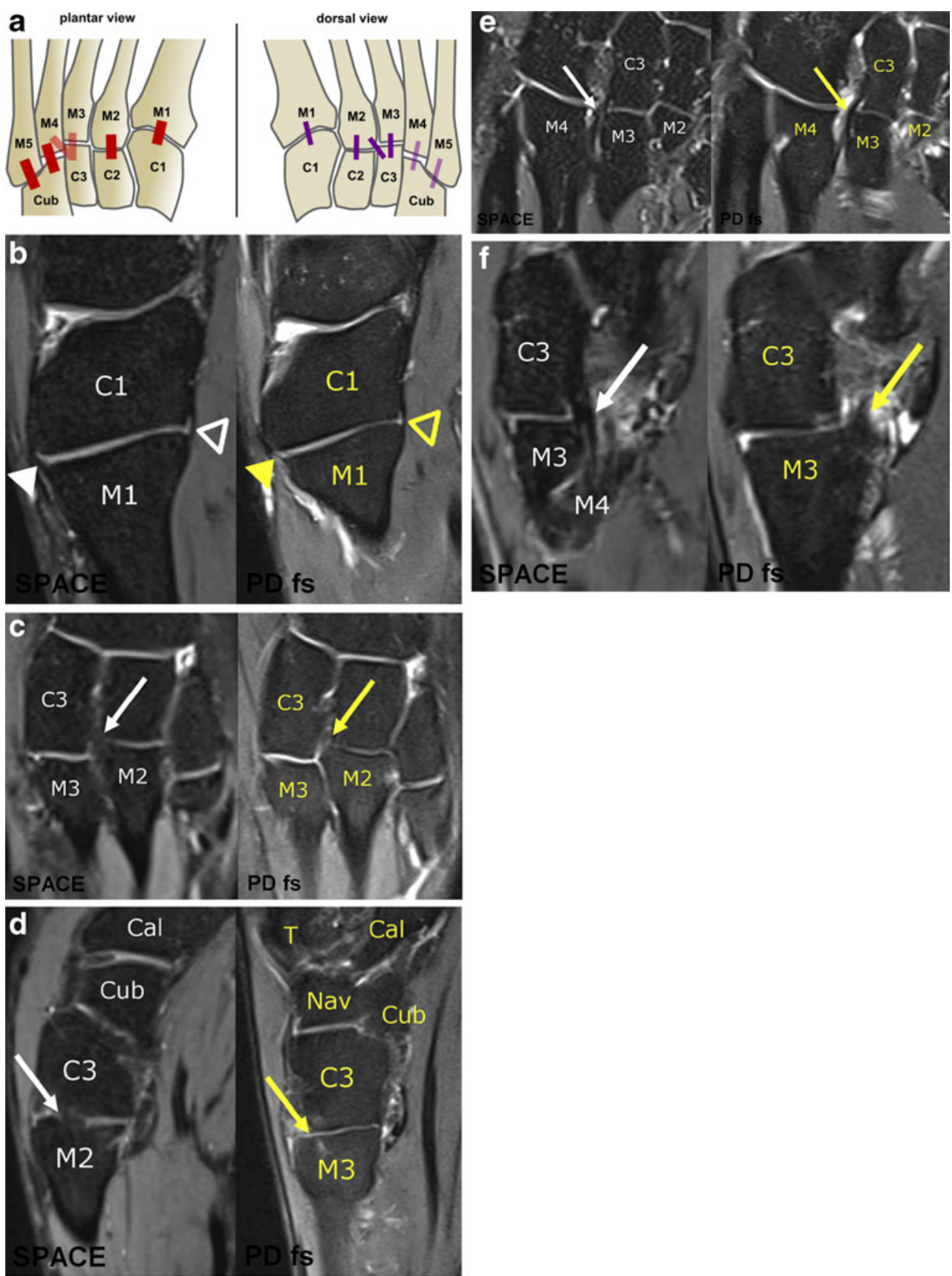

cuboid with the bases of the metatarsals (Fig. 2). The plantar Lisfranc ligament ( $\mathrm{pC} 1-\mathrm{M} 2,3)$ arises from the inferolateral surface of $\mathrm{C} 1$, below the Lisfranc ligament (pC1-M2) insertion and takes an oblique course, oriented laterally and distally. Proximally, it splits into a superficial component that inserts on the base of the third metatarsal and a deeper band that attaches at the base of the second metatarsal on its medial aspect. The other oblique-running plantar TMT ligament ( $\mathrm{pC} 3-\mathrm{M} 3,4)$ originates from the inferolateral surface of the third cuneiform and inserts on the bases of metatarsals 3 and 4 and is inconstant [8].
The thin straight-running dorsal and plantar tarsometatarsal ligaments are defined as capsuloligamentous structures as they cannot be separated from the joint capsules.

4. IMT ligaments: The ten IMT ligaments run horizontally between the bases of the metatarsals with three dorsal, four interosseous, and three plantar IMT ligaments (Fig. 3). The four interosseous IMT ligaments are very short and represent the strongest IMT. No ligament is present between metatarsal 1 and 2 on the dorsal and plantar aspect [8]. 
Fig. 3 Intermetatarsal ligaments. a Diagram illustrating the dorsal (thin blue lines), interosseous (brown lines) and plantar (thick blue lines) intermetatarsal ligaments in a coronal view and the interosseous IMTs also in a transverse view. b Dorsal, interosseous, and plantar IMT, coronal view (SPACE left/PD right). The dorsal IMTs (open arrowheads) have a low signal, the interosseous IMTs (closed arrowheads) a muscleisointense signal and the plantar IMTs (arrows) a low to muscleisointense signal appearance
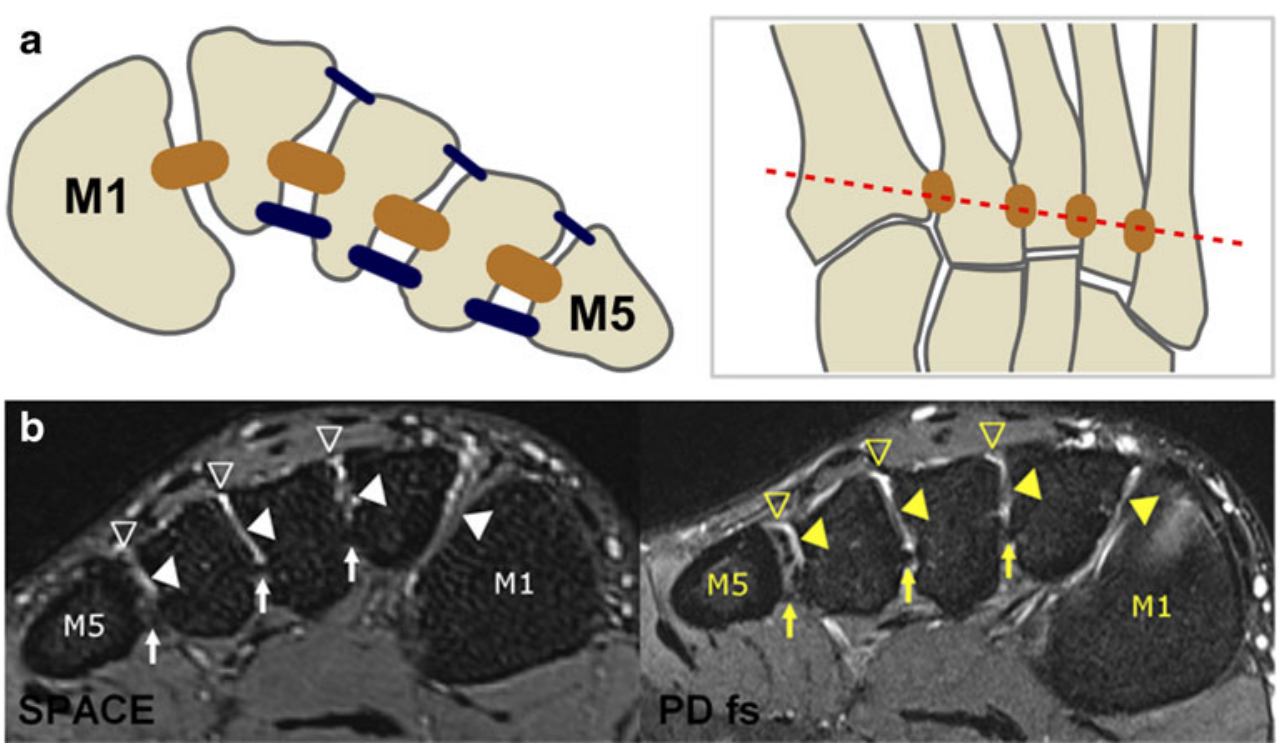

The normal signal characteristic of the 24 ligaments are summarized in Table 2.

\section{Statistical analysis}

Statistical analysis was performed with statistical software (SPSS for Windows, release 17.0; SPSS, Chicago, IL, USA). The detection rate and the appearance score were noted for all ligaments in patients and healthy volunteers. A Chi-square test was used to evaluate differences in the detection rate and the appearance scoring between the 3DSPACE sequence and the PD fs sequences. A difference with $p<0.05$ was considered statistically significant. We compared the point scores of the ligaments in the SPACE sequence with the point scores in the PD fs sequences for all subjects as well as for patients and healthy volunteers separately with a descriptive cross table. The interobserver agreement was determined by calculation of percentage agreement and by a weighted kappa statistics.

\section{Results}

In the 31 asymptomatic feet, 692 ligaments were detected by means of the SPACE sequence, thereof $90.6 \%$ ligaments appeared normal (scores 1 or 2), most ligaments (90.2\%) were completely visible (score 1 ), only $0.4 \%$ were partially visible (score 2). A total of 659 ligaments were detected with the PD fs sequence, $86.6 \%$ ligaments appeared normal (scores 1 or 2$)$, thereof one-third $(28.5 \%$ ) was completely visible (score 1), but $58.1 \%$ ligaments were seen only partially (score 2); $2.4 \%$ ligaments were abnormal in the SPACE sequence, $2 \%$ in the PD fs sequence (score 3 or 4 ); $11.4 \%$ ligaments were absent in the SPACE sequence, $7 \%$ in the PD fs sequence (score 5) (Table 3).
In the 15 symptomatic feet, 327 ligaments were detected by means of the SPACE, $50.6 \%$ ligaments appeared abnormal (score 3). With the PD fs sequence, 308 ligaments were detected, $43.3 \%$ ligaments appeared abnormal (scores 3 or 4). Thereof $17.2 \%$ ligaments were completely visible (score 3 ), and $26.1 \%$ ligaments were only partially visible (score 4). $40.3 \%$ ligaments were normal in the SPACE sequence, $42.2 \%$ in the PD fs sequence (score 1 or 2 ). $14.4 \%$ ligaments were absent in the SPACE sequence, $9.2 \%$ in the PD fs sequence (score 5) (Table 4).

As expected, symptomatic feet had significantly more often abnormal signal alterations of the ligaments than the asymptomatic feet. Eleven out of the 15 patients (symptomatic feet) had ligament strains (= definitely abnormal ligaments; score 3).

In summary, significantly $(p<0.0001)$ more ligaments were seen in the whole length on a single level (score 1 or 3 ) with SPACE (1,011 ligaments) compared to PD fs sequences (323 ligaments) (Tables 3 and 4).

The three Lisfranc-ligaments (dC1-M2, pC1-M2, and $\mathrm{pC} 1-\mathrm{M} 2, \mathrm{M} 3)$, two other oblique-running TMTs (dC3-M2, pC3-M3,4) and the ten IMT ligaments were better seen in the SPACE sequences than in the PD fs sequences. All three Lisfranc ligaments, the oblique-running dorsal TMT (dC3M2) and IMT ligaments were completely visible (score 1 or 3 ) in the SPACE, but only partially (score 2 or 4 ) in the PD fs. The inconstant plantar TMT (pC3-M3,4) was more often visible in the SPACE $(n=33)$ than in the PD fs sequences $(n=4)$ (Tables 3 and 4).

In symptomatic feet, a total of $50.6 \%$ ligaments were definitely abnormal (strain) (score 3) in the SPACE sequence, compared to $17.2 \%$ in the $\mathrm{PD}$ fs sequence, whereas $0 \%$ ligaments were probably abnormal (score 4) in the SPACE sequence, and $26.1 \%$ in the PD fs sequence, respectively. The dorsal TMT ligaments were more often abnormal than the 

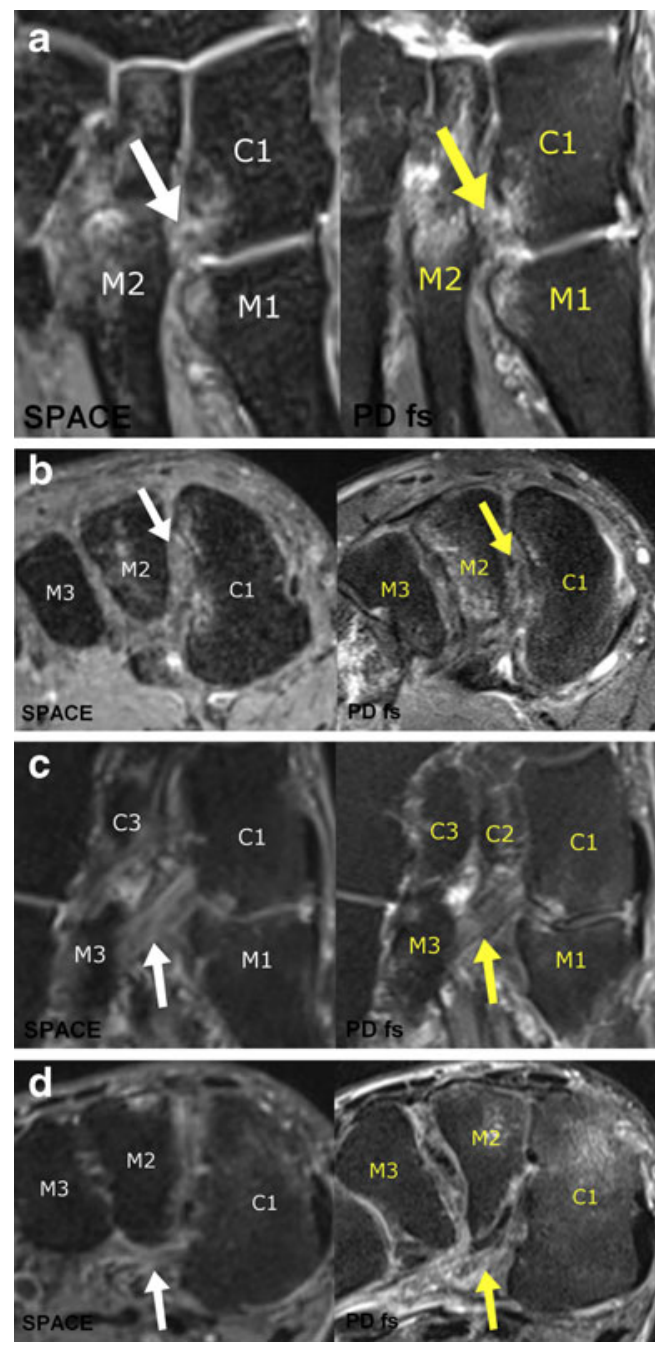

Fig. 4 Examples of patients with abnormal ligaments (ligaments with high signal). a Interosseous Lisfranc ligament (iTMT pC1-M2) strain with high signal, transverse view, with adjacent bone bruise. b Interosseous Lisfranc ligament (iTMT pC1-M2) strain with high signal, coronal view, with adjacent bone bruise. c Plantar Lisfranc ligament (pTMT pC1-M2,3) strain with high signal, transverse view. The ligament connects the plantar side of the first cuneiform with the bases of the second and third metatarsals. d Plantar Lisfranc ligament (pTMT pC1-M2,3) strain with high signal, coronal view. The ligament connects the plantar side of the first cuneiform with the bases of the second and third metatarsals. Note: SPACE sequence is left and PD fs sequence is right in Fig. 4a-d

plantar TMT ligaments (Tables 3 and 4). The most commonly abnormal ligament (11 from 15, on both sequences) was the dorsal TMT ligament between the base of the second metatarsal and the second cuneiform (dC2-M2). The diagnosis of a definitely abnormal ligament (score: 3 ) was easier to make in the SPACE sequence than in the PD fs sequence in the oblique-running ligaments (36 vs. 1).

The interobserver agreement of score 1 to 4 was $66 \%$ ( 239 of 360 possibilities) in the SPACE sequence, and $53 \%$ (192/360) in the PD fs sequence. Regarding the visibility of the ligaments (summary of score 1 and 3 as well as 2 and 4), the agreement was $75 \%(270 / 360)$ in the SPACE sequence, and $58 \%(207 / 360)$ in the PD fs sequence. Regarding the signal intensity of the ligaments (summary of score 1 and 2 as well as 3 and 4), the agreement was $78 \%(280 / 360)$ in the SPACE sequence and $76 \%(272 / 360)$ in the PD fs sequence. In the SPACE sequence, both readers agreed that eight ligaments were absent (score 5), whereas in 28 ligaments only one of the readers defined it as absent (score 5). In the PD fs sequence, both readers agreed in 26 ligaments to be absent (score 5), whereas in 34 ligaments only one of the readers defined it as absent (score 5).

The weighted kappa statistics showed a fair overall interobserver agreement of 0.30 for both the PD fs sequence and the SPACE sequence. The interobserver agreement for the interosseous Lisfranc ligament was good for the SPACE sequence (0.67), while there was only slight agreement for the PD fs sequence (0.20). For the dorsal TMTs, agreement was fair ( 0.36 for SPACE and 0.24 for PD fs), for the plantar TMTs agreement was fair $(0.34$ for SPACE and 0.41 for PD fs), and for the IMTs there was slight agreement for SPACE $(0.20)$ and fair agreement for PD fs $(0.29)$.

\section{Discussion}

The diagnosis of subtle Lisfranc injury may be delayed or missed altogether. Such injuries are predisposed to cause chronic instability, deformity, and pain [12, 27, 28]. Lisfranc joint injuries can be difficult to accurately diagnose because gross lateral deviation or subluxation of the forefoot is less common than subtle midfoot injury. Patients often present with inability to bear weight and swelling in the midfoot region. Examination of the foot reveals forefoot and midfoot edema as well as plantar arch ecchymosis (i.e., plantar ecchymosis sign), which is considered to be characteristic for Lisfranc complex injury [29]. Clinically and radiographically, when noting a diastasis between the first and second ray, the patient is said to have a positive gap sign [30]. In those cases with a clinically and radiographically evident diastasis, sufficient information is provided to make the diagnosis. Subtle ligamentous injuries to the Lisfranc joint are often difficult to detect and go mis- or underdiagnosed. However, proper detection and assessment of those lesions is mandatory in order to apply the correct therapy, which includes nonoperative and operative measures [10-14].

Usually, ligamentous instability can be differentiated into incomplete and complete. The incomplete ligamentous disruption was classified into three groups by Nunley and Vertullo [4]: stage I - ligament sprain without diastasis, stage II - ligament rupture with diastasis of $2-5 \mathrm{~mm}$ between the first and second metatarsals without arch height loss and stage III ligament rupture with diastasis $>5 \mathrm{~mm}$ and loss of midfoot arch height. The incomplete ligamentous disruption 
Table 3 Summary of results of healthy volunteers with asymptomatic feet $(n=31)$

\begin{tabular}{|c|c|c|c|c|c|c|c|c|c|c|}
\hline \multirow[t]{2}{*}{ Ligament groups } & \multirow[t]{2}{*}{ Ligament } & \multirow[t]{2}{*}{ Ligament Abbr. } & \multicolumn{4}{|c|}{$\mathrm{PD}$} & \multicolumn{4}{|c|}{ SPACE } \\
\hline & & & $n$ & $1+2$ & $3+4$ & 5 & $n$ & $1+2$ & $3+4$ & 5 \\
\hline Interosseous TMT & Interosseous Lisfranc lig & iTMT pC1-M2 & 31 & 31 & 0 & 0 & 31 & 31 & 0 & 0 \\
\hline \multirow[t]{7}{*}{ Dorsal TMT } & Dorsal tarsometatarsal lig & dTMT dC1-M1 & 31 & 30 & 1 & 0 & 31 & 30 & 1 & 0 \\
\hline & Dorsal Lisfranc lig & dTMT dC1-M2 & 31 & 31 & 0 & 0 & 31 & 31 & 0 & 0 \\
\hline & Dorsal tarsometatarsal lig & dTMT dC2-M2 & 31 & 30 & 1 & 0 & 31 & 30 & 1 & 0 \\
\hline & Dorsal tarsometatarsal lig & dTMT dC3-M2 & 31 & 31 & 0 & 0 & 31 & 30 & 1 & 0 \\
\hline & Dorsal tarsometatarsal lig & dTMT dC3-M3 & 28 & 27 & 1 & 3 & 28 & 27 & 1 & 3 \\
\hline & Dorsal tarsometatarsal lig & dTMT dCub-M4 & 26 & 26 & 0 & 5 & 28 & 28 & 0 & 3 \\
\hline & Dorsal tarsometatarsal lig & dTMT dCub-M5 & 15 & 14 & 1 & 16 & 20 & 19 & 1 & 11 \\
\hline \multirow[t]{6}{*}{ Plantar TMT } & Plantar tarsometatarsal lig & pTMT pC1-M1 & 31 & 31 & 0 & 0 & 31 & 31 & 0 & 0 \\
\hline & Plantar Lisfranc lig & pTMT pC1-M2,3 & 31 & 31 & 0 & 0 & 31 & 31 & 0 & 0 \\
\hline & Plantar tarsometatarsal lig & pTMT pC2-M2 & 12 & 12 & 0 & 19 & 18 & 18 & 0 & 13 \\
\hline & Plantar tarsometatarsal lig & pTMT pC3-M3,4 & 2 & 2 & 0 & 29 & 23 & 21 & 2 & 8 \\
\hline & Plantar tarsometatarsal lig & pTMT pCub-M4 & 19 & 18 & 1 & 12 & 19 & 18 & 1 & 12 \\
\hline & Plantar tarsometatarsal lig & pTMT pCub-M5 & 30 & 30 & 0 & 1 & 29 & 29 & 0 & 2 \\
\hline \multirow[t]{3}{*}{ Dorsal IMT } & Dorsal intermetatarsal lig & dIMT2-3 & 31 & 30 & 1 & 0 & 31 & 30 & 1 & 0 \\
\hline & Dorsal intermetatarsal lig & dIMT3-4 & 31 & 30 & 1 & 0 & 31 & 30 & 1 & 0 \\
\hline & Dorsal intermetatarsal lig & dIMT4-5 & 31 & 30 & 1 & 0 & 31 & 30 & 1 & 0 \\
\hline Interosseous & Interosseous intermetatarsal lig & iIMT1-2 & 31 & 30 & 1 & 0 & 31 & 30 & 1 & 0 \\
\hline \multirow[t]{3}{*}{ IMT } & Interosseous intermetatarsal lig & iIMT2-3 & 31 & 30 & 1 & 0 & 31 & 30 & 1 & 0 \\
\hline & Interosseous intermetatarsal lig & iIMT3-4 & 31 & 30 & 1 & 0 & 31 & 30 & 1 & 0 \\
\hline & Interosseous intermetatarsal lig & iIMT4-5 & 31 & 30 & 1 & 0 & 31 & 30 & 1 & 0 \\
\hline \multirow[t]{4}{*}{ Plantar IMT } & Plantar intermetatarsal lig & pIMT2-3 & 31 & 30 & 1 & 0 & 31 & 30 & 1 & 0 \\
\hline & Plantar intermetatarsal lig & pIMT3-4 & 31 & 30 & 1 & 0 & 31 & 30 & 1 & 0 \\
\hline & Plantar intermetatarsal lig & pIMT4-5 & 31 & 30 & 1 & 0 & 31 & 30 & 1 & 0 \\
\hline & & Average \% & & 86.6 & 2 & 11 & & 91 & 2.4 & 7 \\
\hline
\end{tabular}

$1=$ completely visible, normal signal

$2=$ partially visible with normal signal

$3=$ completely visible with high signal

$4=$ partially visible with high signal

$5=$ not applicable, absent/not visible

$n=$ total number of visible ligaments

(For a better illustration of normal and abnormal signal of the ligaments score, $1 \& 2$ and $3 \& 4$ were summarized each in one column)

$\mathrm{i}=$ interosseous, $\mathrm{d}=$ dorsal, $\mathrm{p}=$ plantar; TMT $=$ tarsometatarsal, IMT $=$ intermetatarsal; $\mathrm{C} 1=$ first cuneiform, $\mathrm{C} 2=$ second cuneiform, $\mathrm{C} 3=$ third cuneiform, $\mathrm{Cub}=$ cuboid; $\mathrm{M} 1=$ first metatarsal, $\mathrm{M} 2=$ second metatarsal, M3 = third metatarsal, M4 = fourth metatarsal M5 = fifth metatarsal; lig = ligament; $\mathrm{Abbr}=$ abbreviation

group (stage I to III) varies from subtle, nondisplaced injuries that need activity modification only, to partially unstable injuries that need an ORIF (open reduction internal fixation). The therapy of complete ligamentous disruption is controversial as to whether arthrodeses is better than ORIF in any case because of prevalent development of degenerative joint disease after ORIF in the long term [10].

Evaluation of an injured tarsometatarsal joint includes the assessment of dorsal and/or plantar instability. A complete rupture may already be diagnosed clinically by means of a manual stress test (hypermobility of the Lisfranc joint when compared with the healthy side) and easily seen on weightbearing radiographs [28]. Concerning subtle ligamentous injuries, MR imaging can be useful to get information about the extent of injury and the quality of the ligaments. For the surgeon, it is important to know whether the ligament is still attached to the bone, or elongated, massively degenerated, or even absent. In a recent study evaluating the predictive value of MRI for midfoot instability, Raikin et al. [20] found that MRI demonstrating a rupture or grade 2 sprain of the plantar ligament between the first cuneiform and the bases of the second and third MTs is highly predictive of midfoot 
Table 4 Summary of Results of Patients with midfoot pain after trauma $(n=15)$

\begin{tabular}{|c|c|c|c|c|c|c|c|c|c|c|}
\hline \multirow[t]{2}{*}{ Ligament groups } & \multirow[t]{2}{*}{ Ligament } & \multirow[t]{2}{*}{ Ligament Abbr. } & \multicolumn{4}{|l|}{ PD } & \multicolumn{4}{|c|}{ SPACE } \\
\hline & & & $n$ & $1+2$ & $3+4$ & 5 & $n$ & $1+2$ & $3+4$ & 5 \\
\hline Interosseous TMT & Interosseous Lisfranc lig & iTMT pC1-M2 & 15 & 7 & 8 & 0 & 15 & 7 & 8 & 0 \\
\hline \multirow[t]{7}{*}{ Dorsal TMT } & Dorsal tarsometatarsal lig & dTMT dC1-M1 & 15 & 6 & 0 & 0 & 15 & 6 & 9 & 0 \\
\hline & Dorsal Lisfranc lig & dTMT dC1-M2 & 15 & 4 & 11 & 0 & 15 & 7 & 8 & 0 \\
\hline & Dorsal tarsometatarsal lig & dTMT dC2-M2 & 15 & 4 & 0 & 0 & 15 & 4 & 11 & 0 \\
\hline & Dorsal tarsometatarsal lig & dTMT dC3-M2 & 12 & 6 & 9 & 3 & 15 & 6 & 9 & 0 \\
\hline & Dorsal tarsometatarsal lig & dTMT dC3-M3 & 13 & 2 & 2 & 2 & 13 & 3 & 10 & 2 \\
\hline & Dorsal tarsometatarsal lig & dTMT dCub-M4 & 14 & 5 & 1 & 1 & 14 & 5 & 9 & 1 \\
\hline & Dorsal tarsometatarsal lig & dTMT dCub-M5 & 8 & 3 & 7 & 7 & 10 & 4 & 6 & 5 \\
\hline \multirow[t]{6}{*}{ Plantar TMT } & Plantar tarsometatarsal lig & pTMT pC1-M1 & 15 & 6 & 0 & 0 & 15 & 6 & 9 & 0 \\
\hline & Plantar Lisfranc lig & pTMT pC1-M2,3 & 15 & 7 & 8 & 0 & 15 & 7 & 8 & 0 \\
\hline & Plantar tarsometatarsal lig & pTMT pC2-M2 & 7 & 5 & 8 & 8 & 10 & 5 & 5 & 5 \\
\hline & Plantar tarsometatarsal lig & pTMT pC3-M3,4 & 2 & 1 & 13 & 13 & 10 & 7 & 3 & 5 \\
\hline & Plantar tarsometatarsal lig & pTMT pCub-M4 & 4 & 2 & 11 & 11 & 7 & 4 & 3 & 8 \\
\hline & Plantar tarsometatarsal lig & pTMT pCub-M5 & 8 & 4 & 7 & 7 & 8 & 4 & 4 & 7 \\
\hline \multirow[t]{3}{*}{ Dorsal IMT } & Dorsal intermetatarsal lig & dIMT2-3 & 15 & 9 & 6 & 0 & 15 & 7 & 8 & 0 \\
\hline & Dorsal intermetatarsal lig & dIMT3-4 & 15 & 9 & 6 & 0 & 15 & 7 & 8 & 0 \\
\hline & Dorsal intermetatarsal lig & dIMT4-5 & 15 & 9 & 6 & 0 & 15 & 7 & 8 & 0 \\
\hline Interosseous & Interosseous intermetatarsal lig & iIMT1-2 & 15 & 9 & 6 & 0 & 15 & 7 & 8 & 0 \\
\hline \multirow[t]{3}{*}{ IMT } & Interosseous intermetatarsal lig & iIMT2-3 & 15 & 9 & 6 & 0 & 15 & 7 & 8 & 0 \\
\hline & Interosseous intermetatarsal lig & iIMT3-4 & 15 & 9 & 6 & 0 & 15 & 7 & 8 & 0 \\
\hline & Interosseous intermetatarsal lig & iIMT4-5 & 15 & 9 & 6 & 0 & 15 & 7 & 8 & 0 \\
\hline \multirow[t]{4}{*}{ Plantar IMT } & Plantar intermetatarsal lig & pIMT2-3 & 15 & 9 & 6 & 0 & 15 & 7 & 8 & 0 \\
\hline & Plantar intermetatarsal lig & pIMT3-4 & 15 & 9 & 6 & 0 & 15 & 7 & 8 & 0 \\
\hline & Plantar intermetatarsal lig & pIMT4-5 & 15 & 9 & 6 & 0 & 15 & 7 & 8 & 0 \\
\hline & & Average $\%$ & & 42.2 & 43.3 & 14.4 & & 40.3 & 50.6 & 9.2 \\
\hline
\end{tabular}

$1=$ completely visible, normal signal

$2=$ partially visible with normal signal

$3=$ completely visible with high signal

$4=$ partially visible with high signal

$5=$ not applicable, absent/not visible

$n=$ total number of visible ligaments

(For a better illustration of normal and abnormal signal of the ligaments score, $1 \& 2$ and $3 \& 4$ were summarized each in one column)

$\mathrm{i}=$ interosseous, $\mathrm{d}=$ dorsal, $\mathrm{p}=$ plantar; TMT $=$ tarsometatarsal, IMT $=$ intermetatarsal; $\mathrm{C} 1=$ first cuneiform, $\mathrm{C} 2=$ second cuneiform, $\mathrm{C} 3=$ third cuneiform, $\mathrm{Cub}=$ cuboid; $\mathrm{M} 1$ = first metatarsal, M2 = second metatarsal, M3 = third metatarsal, M4 = fourth metatarsal M5 = fifth metatarsal; lig = ligament; Abbr $=$ abbreviation

instability, and these patients should be treated with surgical stabilization.

The current study with a three-dimensional MR sequence (SPACE) and isotropic secondary reformations shows the potential clinical value of a three-dimensional MR sequence. These sequences may help the orthopedic surgeon deciding on the therapy for subtle Lisfranc injuries. Although we were not able to provide surgically confirmed accuracy results, our data indicate that the Lisfranc ligament injuries may be better diagnosed with a three-dimensional MR sequence (SPACE) than with conventional MR sequences based on the higher detection rate, better demarcation, and high interobserver agreement.

The 3D-SPACE sequence features a refocusing pulse train consisting of variable flip-angle pulses of less than $180^{\circ}$. The SPACE sequence acquired on a 3-T scanner combines high spatial resolution (0.5-mm isotropic resolution) and good $\mathrm{T} 2$ contrast within an acceptable examination time (10 min, $22 \mathrm{~s}$ ) by using long echo trains with short echo spacing and high echo train length. The introduction of higher field strengths such as 3T has further improved the diagnostic capability of such sequences in musculoskeletal MRI [31-33]. 
The acquisition time of 3D T2w SPACE in our study was 1 min longer than that of three orthogonal PD fs sequences with acceleration of the image acquisition by parallel imaging. However, the longer acquisition time of the 3D T2w SPACE MR sequence resulted in a higher ligament detection rate.

The SPACE sequence has been applied in several studies assessing other regions of the body where high anatomic resolution is required such as the central nervous system [24], the body trunk [23], the craniocervical junction [22] and the knee $[25,34,35]$. The voxel size of the 3D-SPACE sequence or 3D-FSE-Cube sequence, respectively, for assessment of the craniocervical ligaments was $0.9 \mathrm{~mm}$ [22], for the knee $0.5 \mathrm{~mm}$ [25], and for the ankle $0.6 \mathrm{~mm}$ [26], with comparable detection and visualization of anatomic details to routine 2D-TSE sequences, with the advantage of free 3D reformation.

In summary, the ligaments of the Lisfranc joint are more commonly detectable and better visible with a single 3DSPACE sequence compared to three orthogonal PD fs MR sequences. The intricate anatomy of the ligaments of the Lisfranc joint can be precisely illustrated with the 3DSPACE MR sequence. Thus, we believe that the 3DSPACE MR sequence is a promising MR sequence in the evaluation of normal and pathologic ligaments of the Lisfranc joint.

\section{References}

1. Thompson MC, Mormino MA. Injury to the tarsometatarsal joint complex. J Am Acad Orthop Surg. 2003;11(4):260-7.

2. Curtis MJ, Myerson M, Szura B. Tarsometatarsal joint injuries in the athlete. Am J Sports Med. 1993;21(4):497-502.

3. Norfray JF, Geline RA, Steinberg RI, Galinski AW, Gilula LA. Subtleties of Lisfranc fracture-dislocations. AJR Am J Roentgenol. 1981;137(6):1151-6.

4. Nunley JA, Vertullo CJ. Classification, investigation, and management of midfoot sprains: Lisfranc injuries in the athlete. Am J Sports Med. 2002;30(6):871-8.

5. Shapiro MS, Wascher DC, Finerman GA. Rupture of Lisfranc's ligament in athletes. Am J Sports Med. 1994;22(5):687-91.

6. de Palma L, Santucci A, Sabetta SP, Rapali S. Anatomy of the Lisfranc joint complex. Foot Ankle Int. 1997;18(6):356-64.

7. Preidler KW, Wang YC, Brossmann J, Trudell D, Daenen B, Resnick D. Tarsometatarsal joint: anatomic details on MR images. Radiology. 1996;199(3):733-6.

8. Sarrafian SK. Anatomy of the foot and ankle: descriptive, topographic, functional. 2nd ed. Philadelphia: J.B. Lippincott; 1993.

9. The lower limb. In: Moore KL, ed. Clinically oriented anatomy. 2nd ed. Baltimore, MD: Williams \& Wilkins; 1985:471.

10. Coetzee JC. Making sense of Lisfranc injuries. Foot Ankle Clin. 2008;13(4):695-704. ix.

11. Henning JA, Jones CB, Sietsema DL, Bohay DR, Anderson JG. Open reduction internal fixation versus primary arthrodesis for Lisfranc injuries: a prospective randomized study. Foot Ankle Int. 2009;30(10):913-22.
12. Ly TV, Coetzee JC. Treatment of primarily ligamentous Lisfranc joint injuries: primary arthrodesis compared with open reduction and internal fixation. A prospective, randomized study. J Bone Joint Surg Am. 2006;88(3):514-20.

13. Myerson MS, Cerrato RA. Current management of tarsometatarsal injuries in the athlete. J Bone Joint Surg Am. 2008;90(11):252233.

14. Watson TS, Shurnas PS, Denker J. Treatment of Lisfranc joint injury: current concepts. J Am Acad Orthop Surg. 2010;18 (12):718-28.

15. Castro M, Melao L, Canella C, Weber M, Negrao P, Trudell D, et al. Lisfranc joint ligamentous complex: MRI with anatomic correlation in cadavers. AJR Am J Roentgenol. 2010;195(6):W447-455.

16. Delfaut EM, Rosenberg ZS, Demondion X. Malalignment at the Lisfranc joint: MR features in asymptomatic patients and cadaveric specimens. Skeletal Radiol. 2002;31(9):499-504.

17. Macmahon PJ, Dheer S, Raikin SM, Elias I, Morrison WB, Kavanagh $\mathrm{EC}$, et al. MRI of injuries to the first interosseous cuneometatarsal (Lisfranc) ligament. Skeletal Radiol. 2009;38(3):255-60.

18. Potter HG, Deland JT, Gusmer PB, Carson E, Warren RF. Magnetic resonance imaging of the Lisfranc ligament of the foot. Foot Ankle Int. 1998;19(7):438-46.

19. Preidler KW, Brossmann J, Daenen B, Goodwin D, Schweitzer M, Resnick D. MR imaging of the tarsometatarsal joint: analysis of injuries in 11 patients. AJR Am J Roentgenol. 1996;167(5):1217-22.

20. Raikin SM, Elias I, Dheer S, Besser MP, Morrison WB, Zoga AC. Prediction of midfoot instability in the subtle Lisfranc injury. Comparison of magnetic resonance imaging with intraoperative findings. J Bone Joint Surg Am. 2009;91(4):892-9.

21. Rand T, Frank L, Pretterklieber M, Muhle C, Resnick D. Intertarsal ligaments: high resolution MRI and anatomic correlation. J Comput Assist Tomogr. 2000;24(4):584-93.

22. Baumert B, Wortler K, Steffinger D, Schmidt GP, Reiser MF, Baur-Melnyk A. Assessment of the internal craniocervical ligaments with a new magnetic resonance imaging sequence: threedimensional turbo spin echo with variable flip-angle distribution (SPACE). Magn Reson Imaging. 2009;27(7):954-60.

23. Lichy MP, Wietek BM, Mugler 3rd JP, Horger W, Menzel MI, Anastasiadis A, et al. Magnetic resonance imaging of the body trunk using a single-slab, 3-dimensional, T2-weighted turbo-spinecho sequence with high sampling efficiency (SPACE) for high spatial resolution imaging: initial clinical experiences. Invest Radiol. 2005;40(12):754-60.

24. Mugler 3rd JP, Bao S, Mulkern RV, Guttmann CR, Robertson RL, Jolesz FA, et al. Optimized single-slab three-dimensional spinecho MR imaging of the brain. Radiology. 2000;216(3):891-9.

25. Notohamiprodjo M, Horng A, Pietschmann MF, Muller PE, Horger W, Park J, et al. MRI of the knee at $3 \mathrm{~T}$ : first clinical results with an isotropic PDfs-weighted 3D-TSE-sequence. Invest Radiol. 2009;44(9):585-97.

26. Stevens KJ, Busse RF, Han E, Brau AC, Beatty PJ, Beaulieu CF, et al. Ankle: isotropic MR imaging with 3D-FSE-cube-initial experience in healthy volunteers. Radiology. 2008;249(3):1026-33.

27. Goossens M, De Stoop N. Lisfranc's fracture-dislocations: etiology, radiology, and results of treatment. A review of 20 cases. Clin Orthop Relat Res. 1983(176):154-162.

28. Faciszewski T, Burks RT, Manaster BJ. Subtle injuries of the Lisfranc joint. J Bone Joint Surg Am. 1990;72(10):1519-22.

29. Ross G, Cronin R, Hauzenblas J, Juliano P. Plantar ecchymosis sign: a clinical aid to diagnosis of occult Lisfranc tarsometatarsal injuries. J Orthop Trauma. 1996;10(2):119-22.

30. Davies MS, Saxby TS. Intercuneiform instability and the "gap" sign. Foot Ankle Int. 1999;20(9):606-9.

31. Mosher TJ. Musculoskeletal imaging at $3 \mathrm{~T}$ : current techniques and future applications. Magn Reson Imaging Clin N Am. 2006;14 (1):63-76. 
32. Ramnath RR. 3 T MR imaging of the musculoskeletal system (Part II): clinical applications. Magn Reson Imaging Clin N Am. 2006;14(1):41-62.

33. Ramnath RR. 3 T MR imaging of the musculoskeletal system (Part I): considerations, coils, and challenges. Magn Reson Imaging Clin N Am. 2006;14(1):27-40.

34. Kijowski R, Davis KW, Woods MA, Lindstrom MJ, De Smet AA, Gold GE, et al. Knee joint: comprehensive assessment with 3D isotropic resolution fast spin-echo MR imaging-diagnostic performance compared with that of conventional MR imaging at 3.0 T. Radiology. 2009;252(2):486-95.

35. Ristow O, Stehling C, Krug R, Steinbach L, Sabo G, Ambekar A, et al. Isotropic 3-dimensional fast spin echo imaging versus standard 2-dimensional imaging at 3.0 T of the knee: artificial cartilage and meniscal lesions in a porcine model. J Comput Assist Tomogr. 2010;34(2):260-9. 